\title{
How Many Templates for GW Chirp Detection? The Minimal-Match Issue Revisited
}

\author{
R. P. Croce, Th. Demma, V. Pierro, and I. M. Pinto \\ Wavesgroup, University of Sannio at Benevento, Italy \\ M. Longo, S. Marano and V. Matta \\ D.I. ${ }^{3}$ E., University of Salerno, Italy
}

\begin{abstract}
In a recent paper dealing with maximum likelihood detection of gravitational wave chirps from coalescing binaries with unknown parameters we introduced an accurate representation of the nosignal cumulative distribution of the supremum of the whole correlator bank. This result can be used to derive a refined estimate of the number of templates yielding the best tradeoff between detector's performance (in terms of lost signals among those potentially detectable) and computational burden.
\end{abstract}

\section{INTRODUCTION}

Optimizing the design of the correlator-bank for detecting gravitational wave (henceforth GW) chirps from coalescing binaries (henceforth $\mathrm{CB}$ ) with unknown parameters is a key and yet not settled problem. The main goal is to identify and adopt the best tradeoff between detector's performance and computational cost. In a recent paper [1] we introduced an accurate representation of the cumulative distribution function (henceforth CDF) of the supremum of a bank of correlators in the null hypothesis taking into account the nonzero covariance among them, extending previous results by Mohanty [2]. These findings can be used to re-address the following questions: i) whether it really makes sense to use the largest number of correlators compatible with the available computing power, and ii) whether the simple argument [3] whereby the fraction of potentially observable sources which would be lost as an effect of template sparsity is $\left(1-\Gamma^{3}\right), \Gamma$ being the minimal match [4] is still valid when one takes into account both the dependence of the detection threshold on the total number of correlators, and the covariance among the correlators, at a given false-alarm level. The above issues are discussed in Sect. 3 and 4 below, while Sect. 2 is aimed at introducing the relevant notation.

\section{BACKGROUND}

The maximum likelihood (henceforth ML) strategy for detecting GW chirps from CBs with unknown parameters goes through the following steps. First, a set of $N_{\Delta}$ reduced templates $[\underline{8}]$ is set up, viz.:

$$
T\left(f, \vec{\xi}_{T}\right)=T_{0} f^{-7 / 6} \exp \left[j \psi\left(f, \vec{\xi}_{T}\right)\right],
$$

where $\psi\left(f, \vec{\xi}_{T}\right)$ is the (post-newtonian) chirp phasing function [5], $\vec{\xi}_{T}$ represents the intrinsic source parameters (binary companion masses and spin parameters), and $T_{0}$ is a suitable constant. Second, a set of non-coherent reduced correlators

$$
C[A, T]=\sup _{\tau}\left|\mathcal{F}_{f \rightarrow \tau} K_{A}\left[f, \vec{\xi}_{T}\right]\right|
$$

is computed, where $\mathcal{F}$ is the Fourier-transform operator and:

$$
K_{A}\left[f, \vec{\xi}_{T}\right]=\left\{\begin{array}{l}
2 \frac{A(f) T^{*}\left(f, \vec{\xi}_{T}\right)}{\Pi(f)}, \quad f \in\left(f_{i}, f_{s}\right) \\
0, \quad \text { elsewhere. }
\end{array}\right.
$$

In eq. (2.3) $A(f)=S(f)+N(f)$ are the (spectral, noisy) data, resulting from the superposition of a (possibly null) signal $S(f)$ and a realization $N(f)$ of the antenna noise, $\left(f_{i}, f_{s}\right)$ is the antenna spectral window, and $\Pi(f)$ is the (one-sided) antenna noise power spectral density (henceforth PSD). The Fourier transform in (2.2) is implemented using a discrete finite algorithm (DFT). The corresponding number of coalescency times will be henceforth denoted by $N_{\Theta}$; each reduced template is thus used to compute $N_{\Theta}$ (non-reduced) correlators.

Once all correlators have been evaluated, the final step consists in comparing the supremum

$$
\Lambda=\sup _{\vec{\xi}_{T}} C[A, T]
$$


(or any nondecreasing function thereof) to a threshold $\gamma$ depending on the prescribed false-alarm probability

$$
\alpha=\operatorname{prob}[\Lambda>\gamma \mid \text { no signal }] \text {. }
$$

The threshold $\gamma$ can be accordingly computed from the no-signal cumulative distribution of $\Lambda$. Whenever $\Lambda>\gamma$ one declares that a signal has been observed (within false alarm probability $\alpha$ ), and estimates the unknown source parameters from those of the template yielding the largest correlator.

On the other hand, it can be shown that the false-dismissal probability

$$
\beta=\operatorname{prob}[\Lambda \leq \gamma \mid \text { nonzero signal }]
$$

is a decreasing function of the match

$$
M[S, T]=\left(\int_{f_{i}}^{f_{s}} f^{-7 / 3} \frac{d f}{\Pi(f)}\right)^{-1} \sup _{\tau}\left|\mathcal{F}_{f \rightarrow \tau} K_{S}\left[f, \vec{\xi}_{T}\right]\right|,
$$

between the signal $S$ and the (most similar available) template $T$, where:

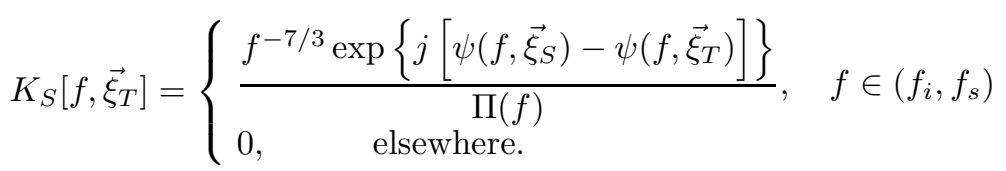

The match is a measure of the similarity between $S$ and $T$, and is obviously equal to one iff $S=T$. The reducedtemplate set should be accordingly designed so as to cope with the more or less obvious prescription

$$
\forall S \in \mathcal{S}, \exists T \in \mathcal{L}: M[S, T] \geq \Gamma
$$

where $\mathcal{S}$ and $\mathcal{L}$ are the allowed signal and template sets, respectively, and $\Gamma$ is the minimal match [4].

\section{MINIMAL MATCH VS. NUMBER OF TEMPLATES}

The natural question is how many reduced templates one should use in practice. The standard argument suggests using the largest total number $N_{\Delta}$ of (reduced) templates in the allowed (reduced) parameter-space search subset, compatible with the available computing power, so as to achieve the largest-minimal-match $\Gamma$ over the class of admissible sources.

In this connection, it should be stressed first that a visible knee point in the $\Gamma$ vs. $N_{\Delta}$ curve invariably exists at some value $N_{\Delta}=N_{*}$, beyond which any further increase in $N_{\Delta}$ yields only a modest increase in $\Gamma$.

This is illustrated in Fig.1 for the simplest case of the one-parameter family of (reduced) newtonian waveforms in the range of chirp masses $\left(0.2 M_{\odot} \leq \mathcal{M} \leq 10 M_{\odot}\right)$. To compute the curve in Fig. 1 we used the (initial) LIGO noise PSD 6]

$$
\Pi(f)=\frac{1}{4}\left\{1+\left(\frac{f_{0}}{f}\right)^{4}+2\left(\frac{f}{f_{0}}\right)^{2}\right\},
$$

with $f_{0}=300 \mathrm{~Hz}, f_{i}=40 \mathrm{~Hz}$ and $f_{s}=400 \mathrm{~Hz}$; we sampled the data at $3.2 \mathrm{KHz}$ (4-times the Nyquist rate), and let $N_{\Theta}=2^{21}$, which corresponds to the longest-lived source allowed $\left(\mathcal{M}=0.2 M_{\odot}\right)$.

A knee point separating intervals of different (almost constant) slope in the $\Gamma$ versus $N_{\Delta}$ curve occurs in Fig. 1 at $N_{\Delta} \approx 5 \cdot 10^{3}$. Except for the numerical value of $N_{*}$, the existence of the above knee point is a generic feature of the $\Gamma$ vs. $N_{\Delta}$ curve, occurring also for more realistic, higher-order post-newtonian waveforms and templates.

\section{UNDETECTED OBSERVABLE SOURCES VS. NUMBER OF TEMPLATES}

It was first argued in [3] that (under the simplest assumption of isotropically and homogeneously distributed sources) the fraction of potentially observable sources which would be undetected as an effect of template mismatch is $\left(1-\Gamma^{3}\right)$. This argument conceals an obvious naivety insofar as it neglects the dependence of the detection threshold $\gamma$ corresponding to a given false-alarm probability $\alpha$ on the total number of templates $N=N_{\Theta} N_{\Delta}$, and hence on $\Gamma$ itself. 
In order to gain insight into this issue, it is expedient to work in terms of the detection probability $P_{d}$, and capitalize on the accurate approximate representations for the no-signal cumulative distribution of the whole-bank supremum obtained in [1], which is needed to compute the threshold $\gamma$ corresponding to a given $\alpha$.

Accordingly, let

$$
P_{d}=1-\beta=1-Q[\gamma-E(\Lambda)]
$$

where $Q(\cdot)$ is an unknown CDF, whose density can be nonetheless safely assumed as being unimodal and centersymmetric, at least for meaningful values of the signal-to-noise ratio. In the worst case of minimal match,

$$
E(\Lambda) \approx \Gamma d
$$

where $d$ is the signal deflection (signal-to-noise ratio), viz.:

$$
d:=\left|2 \int_{f_{i}}^{f_{s}} \frac{S(f) S^{*}(f)}{\Pi(f)} d f\right|^{1 / 2}
$$

and we assume $d \gg 1$. Let the observable sources be those for which $P_{d} \geq 0.5$. Under the above assumptions for $Q(\cdot)$, these correspond to:

$$
d \geq \frac{\gamma}{\Gamma}
$$

The deflection $d$ on the other hand can be written

$$
d=\frac{K}{R}
$$

where $R$ is the source distance, and $K$ is a constant depending on the antenna and source features, as well as their mutual orientation. Equation (4.4) allows to define the radius $R$ of the antenna-centered sphere which contains all sources of a given class (seen by the same antenna, sharing the same features and orientation) producing a deflection $d \geq \gamma($ i.e., observable), viz.:

$$
R=\frac{K \Gamma}{\gamma}
$$

Hence the number of observable sources in the given class, under the most obvious hypothesis of uniform (homogeneous and isotropic) spatial source density $\rho_{s}$ is:

$$
N_{s}=\frac{4}{3} \pi \rho_{s}\left[\frac{K \Gamma}{\gamma}\right]^{3} .
$$

Letting $\Gamma_{\max }, N_{\max }$ the largest achievable minimal match, and the related number of templates, and denoting as $N_{s}^{(\max )}$ the corresponding value of $N_{s}$, one has from (4.7):

$$
\eta=\frac{N_{s}}{N_{s}^{(\max )}}=\left(\frac{\Gamma}{\Gamma_{\max }}\right)^{3}\left[\frac{\gamma\left(\alpha, N_{\max }\right)}{\gamma(\alpha, N)}\right]^{3}
$$

where the dependence of $\gamma$ from $\alpha$ and $N$ has been written explicitly. The quantity $1-\eta$ represents the fraction of observable sources which would be lost due to poor minimal match $\left(\Gamma<\Gamma_{\max }\right)$. Note that the ratio $\eta$ does $n o t$ depend on $K$.

Neglecting the dependance of $\gamma$ on $N$ (and hence on $\Gamma$ ), the second factor on the r.h.s. of (4.8) cancels, and we are left with

$$
\eta=\left(\frac{\Gamma}{\Gamma_{\max }}\right)^{3}:=\eta_{0}
$$

which does not depend on $\alpha$. Under the same assumption, $N_{s}$ in (4.7) increases monotically with $\Gamma$, attaining its supremum $N_{s}^{(\max )}$ at $\Gamma_{\max }=1$, yielding $\eta_{0}=\Gamma^{3}$. This is, in essence, the simplest argument introduced in $\underline{3}$.

In order to refine this reasoning one should obviously include the dependence of $\gamma$ on $N$, which in turn is affected by the covariance among the correlators [1]. For illustrative purposes, we shall again stick at the simplest case of 
newtonian signals and templates, using the same parameters as in Fig. 1, and use the best available approximant for the no-signal CDF of the detection statistic $\Lambda$ from 1] in order to compute the ratio $\gamma\left(\alpha, N_{\max }\right) / \gamma(\alpha, N)$ on the r.h.s. of (4.8) 9]. In Fig. 2 we accordingly compare $\eta_{0}$ (eq. (4.9), dashed line), to $\eta$ (eq. (4.8), solid lines), for $\alpha=10^{-k}, k=2,3,4$ (top-to bottom), as functions of $N_{\Delta}$. In Fig. 2 we assumed $\Gamma_{\max }=0.99$ as a bona fide practical limit value, and computed $N_{\max }$ accordingly. From Fig. 2 it is seen that a knee-point in the curves exists at $N_{\Delta} \approx N_{*} \approx 5 \cdot 10^{3}$. It is also seen that $\eta$ exceeds by a non-negligible $5 \%$ typ. the plain estimate $\eta_{0}$, for $N_{\Delta} \approx N_{*}$, if the best available approximant for the no-signal CDF of the detection statistic $\Lambda$ is used to compute the threshold 10 . It is also interesting to compare in terms of $\eta$ alternative (less accurate, i.e., yielding larger thresholds for the same $\alpha$ and $N$ ) approximants for the no-signal CDF of the detection statistic $\Lambda$. This is obtained by computing $N_{s}^{(m a x)}$ using the best available approximant, i.e., using this latter to compute $\gamma\left(\alpha, N_{\max }\right)$ in (4.8). The resulting curves are shown in Fig. 3, where again we assumed $\Gamma_{\max }=0.99$ as a bona fide practical limit-value, and computed $N_{\max }$ accordingly. Specifically, the (scaled) quantities $\eta^{(0,1,2)}$ in Fig. 3 correspond to ignoring the covariance among the correlators (dotted line), and to including the covariance among nearest-neighbour correlators along one (coalescency-time, dashed line) or both (coalescency-time and chirp-mass, solid line, best available approximant) coordinates of the newtonian parameter-space, respectively 1]. For each case, the $\eta$ vs. $N_{\Delta}$ curves corresponding to $\alpha=10^{-2}, 10^{-3}, 10^{-4}$ are displayed. It is seen that the dependence of $\eta$ on $\alpha$ becomes the less relevant, the better approximant is used.

\section{CONCLUSIONS}

On the basis of the above findings, the following conclusions can be drawn. Inspection of Fig.s 1 and 2 , shows a consistent evidence that increasing the number of templates beyond a critical value corresponding to the knee-point in the curve of $\Gamma$ vs. $N_{\Delta}$ (Fig. 1), does not produce a sensible increase in the detectable fraction of potentially observable sources, at the expense of a marked growth of computational load. On the other hand, using the presently most accurate available representation of the no-signal cumulative distribution of the (whole-bank) detection statistic to compute the threshold results in a sizeable increase $(\geq 5 \%)$ in the detectable fraction of potentially observable sources over the naive $\propto \Gamma^{3}$ estimate.

Both findings above support the current trend [7] toward the use of hierarchical search strategies for best tradeoff between detector's performance and computational burden.

\section{Acknowledgements}

This work has been sponsored in part by the European Community through a Senior Visiting Scientist Grant to I.M. Pinto at NAO - Spacetime Astronomy Division, Tokyo, Japan, in connection with the TAMA project. I.M. Pinto wishes to thank all the TAMA staff at NAO, and in particular prof. Fujimoto Masa-Katsu and prof. Kawamura Seiji for kind hospitality and stimulating discussions.

\section{References}

[1] R.P. Croce et al., Class. Quantum Grav. 20 (2003) S803.

[2] S.D. Mohanty, Phys Rev. D57 (1998) 630.

[3] Apostolatos Th. Phys. Rev. D54 (1996) 2421.

[4] B.J. Owen Phys. Rev. D53 (1996) 6749.

[5] B. Owen and B.S. Sathyaprakash, Phys. Rev. D60 (1999) 022002.

[6] L.S. Finn and D. Chernoff, Phys. Rev. D47 (1993) 2198.

[7] S. D. Mohanty and S. V. Dhurandhar, Phys. Rev. D 54 (1996) 7108.

[8] Reduced chirp templates are obtained by setting the template coalescing time and coalescing phase both equal to zero.

[9] Note that $\gamma\left(\alpha, N_{\max }\right) / \gamma(\alpha, N) \geq 1$, since $\gamma(\alpha, N)$ is a non-decreasing function of $N$, whatever $\alpha$.

[10] Note that the referred approximant is still a lower bound for the true (unknown) cumulative distribution [1], which accordingly provides a conservative estimate of the threshold. 


\section{Captions to the Figures}

Figure 1 - Minimal match vs. number of reduced templates in $0.2 M_{\odot} \leq \mathcal{M} \leq 10 M_{\odot}$. Newtonian waveforms, Ligo-I noise.

Figure 2 - Detectable fraction of potentially observable sources, in $0.2 M_{\odot} \leq \mathcal{M} \leq 10 M_{\odot} \cdot \eta_{0}$ (eq. 4.9), dashed line) and $\eta$ (eq. (4.8), solid lines) vs. $N_{\Delta}$, for $\alpha=10^{-k}, k=2,3,4$ (top-to bottom). Newtonian waveforms, Ligo-I noise.

Figure 3 - Detectable fraction of potentially observable sources, with $0.2 M_{\odot} \leq \mathcal{M} \leq 10 M_{\odot}$ vs. number of reduced templates at different false-alarm levels, using different models of the no-signal cumulative distribution of the detection statistic. Newtonian waveforms, Ligo-I noise. 


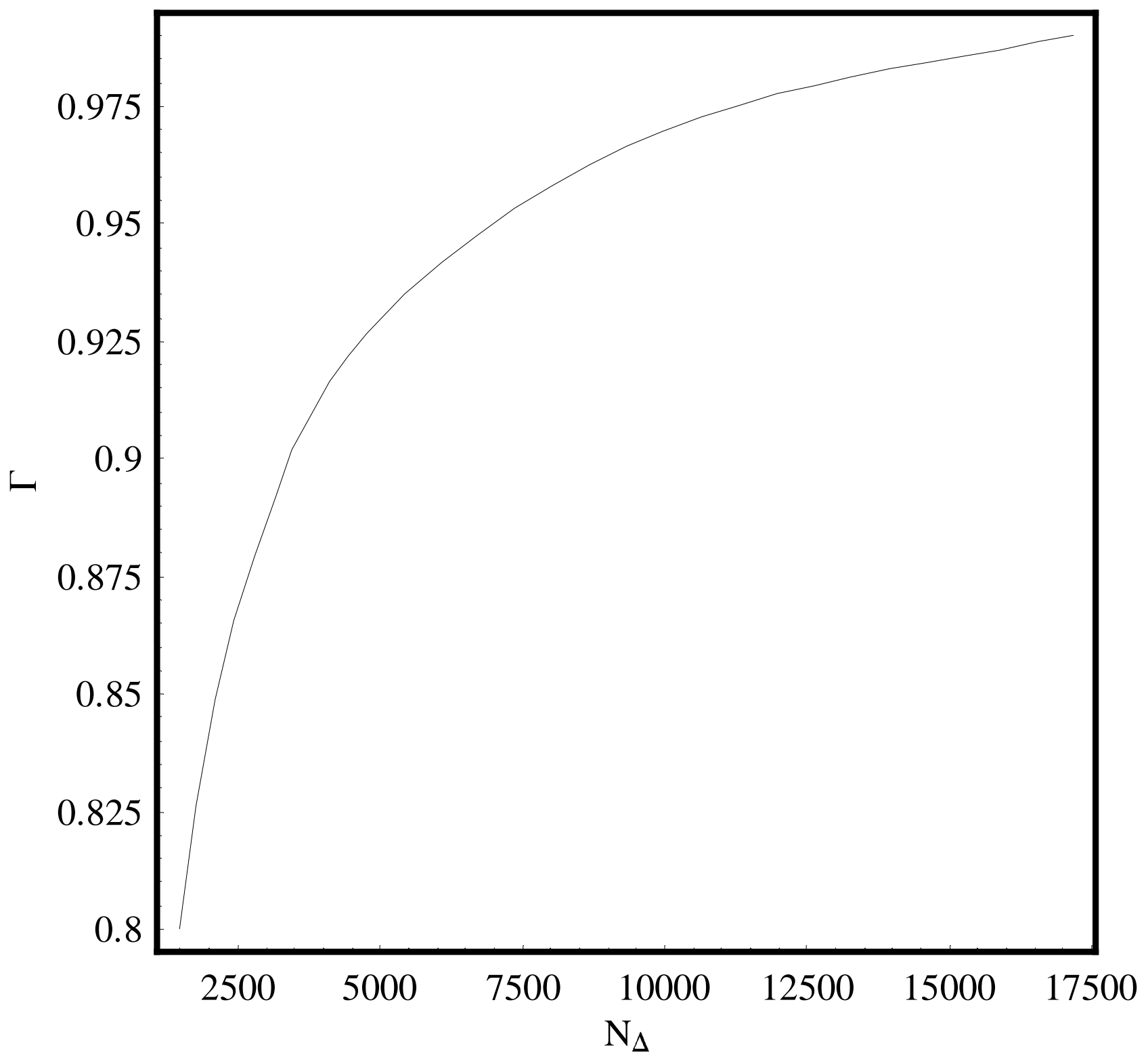




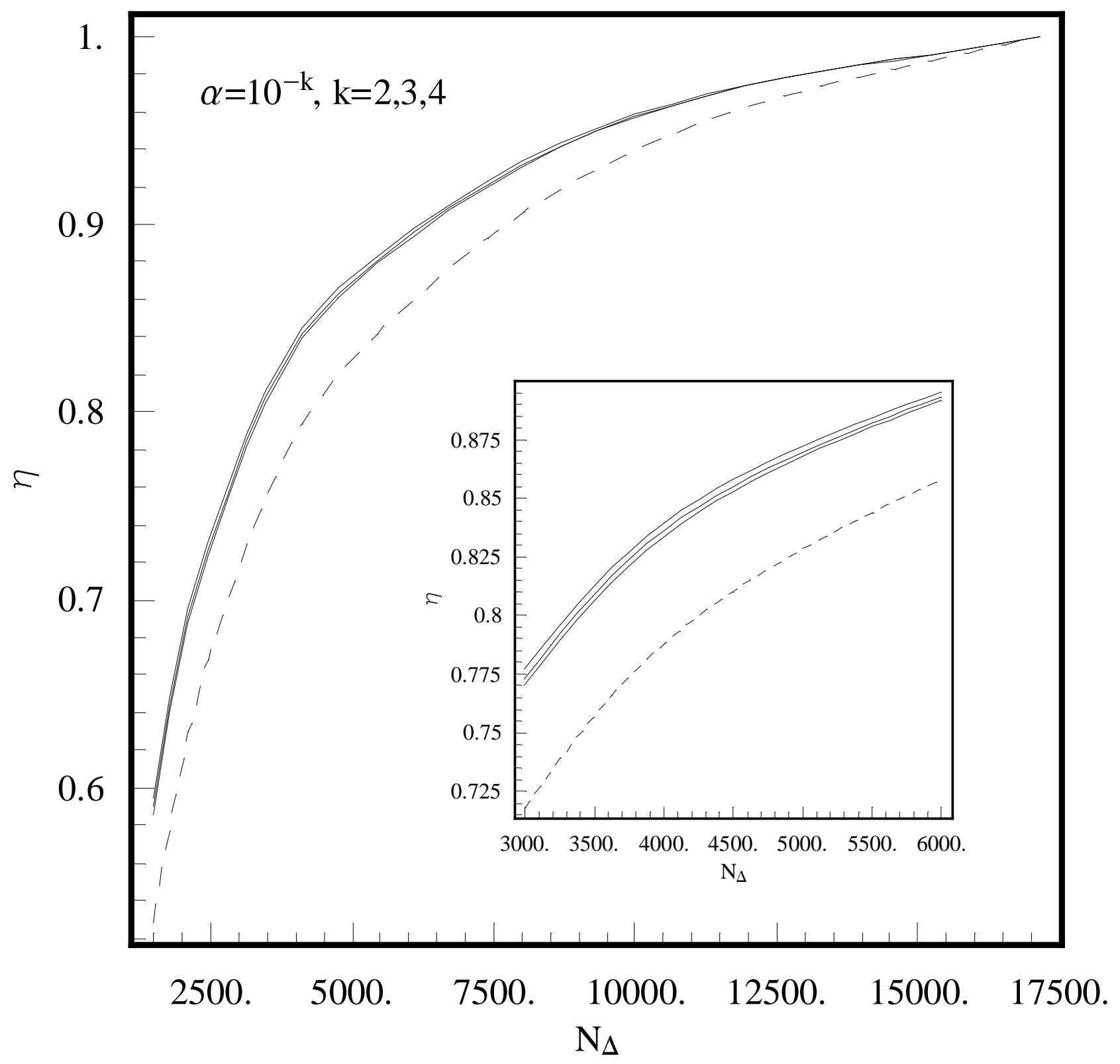




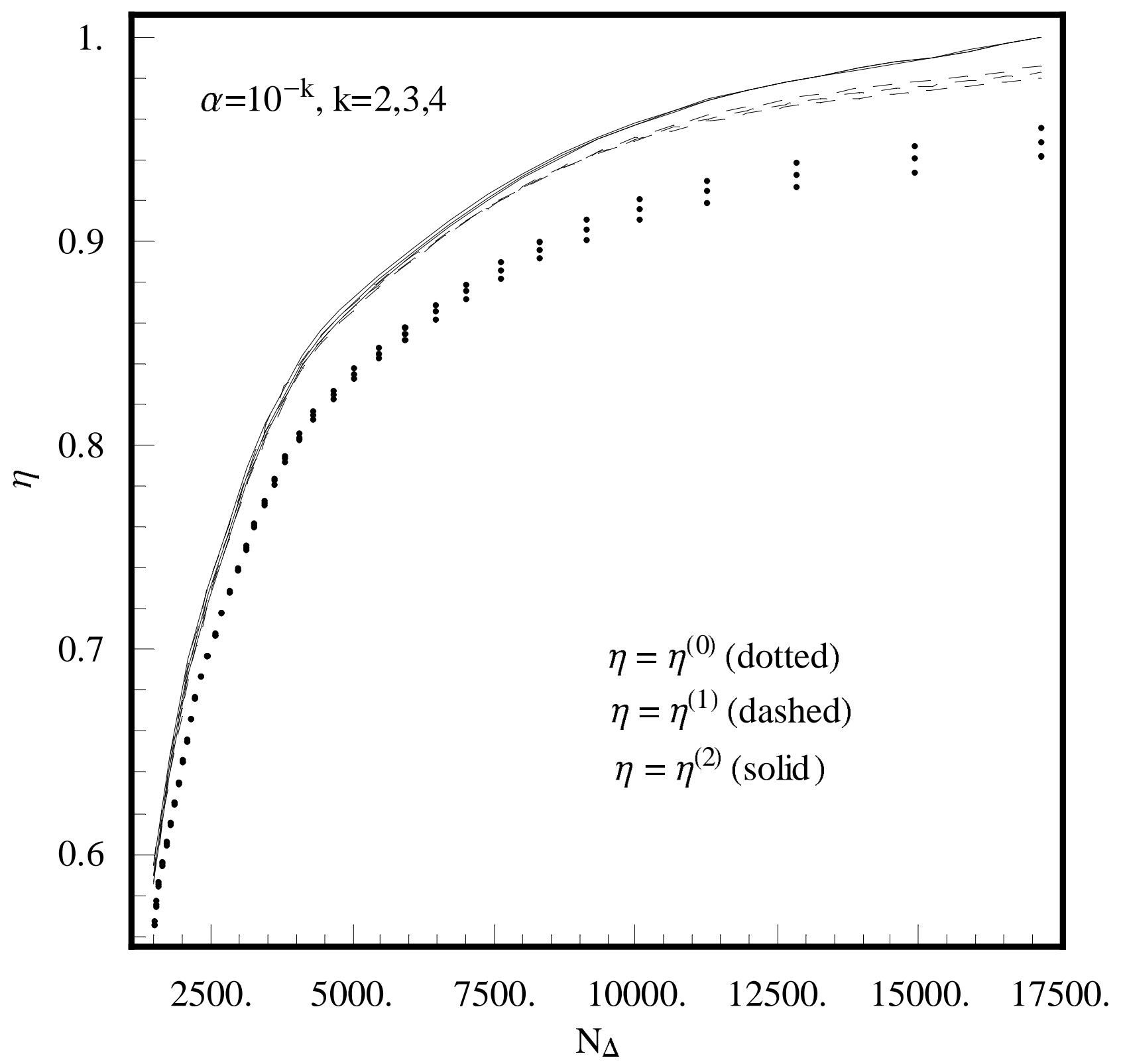

